\title{
Stirring the quantum vacuum: Angular Casimir momentum of a Landau charge
}

\author{
B. A. van Tiggelen* \\ Université Grenoble Alpes, CNRS, LPMMC, 38000 Grenoble, France
}

(Received 16 September 2019; published 21 November 2019)

\begin{abstract}
We consider the angular momentum of a charge $q$ rotating in a homogeneous magnetic field and study the role of the electromagnetic quantum vacuum. Its orbital angular momentum is caused by the recoil of energetic vacuum photons that grows as $n^{2}$, i.e., faster than the kinetic angular momentum $-2 n \hbar$ of a Landau level.
\end{abstract}

DOI: 10.1103/PhysRevResearch.1.033118

\section{INTRODUCTION}

The radiation of the electromagnetic (EM) quantum vacuum is perfectly isotropic and possesses an energy density $\hbar \omega d \omega^{3} / \pi^{3} c_{0}^{3}$ in the frequency interval $d \omega$. This statement is Lorentz invariant [1]. The mystery of its UV divergence is still one of the major challenges in physics, but in general poses no problems in calculating the Casimir force between dielectric or metallic objects [2]. Due to its perfect isotropy, the energy current and momentum density of the quantum vacuum vanish.

This is no longer true when the vacuum interacts with matter. In a pioneering work, Feigel predicted the momentum of the quantum vacuum in bianisotropic materials [3]. In such materials, the index of refraction achieves a contribution proportional to $g(\omega) \mathbf{k} \cdot \mathbf{S}$, with $\mathbf{k}$ the wave vector and $\mathbf{S}$ some vector with the symmetry of momentum. Although the Poynting vector of the quantum vacuum still vanishes for such media [4], a momentum density emerges that suffers from a divergence at high energies. Any small time variation of this momentum would give rise to a Casimir-type force, and is thus measurable. Nevertheless, this force is fundamentally different from the usual Casimir and Casimir-Polder forces [2], which are both free from high-energy divergences and are essentially caused by low-energy vacuum photons. In fact, the Casimir momentum discussed in Refs. [3,4] is much more akin to the Lamb shift in atomic energy levels, determined by relativistic photons of the quantum vacuum [1], and is thus better referred to as Lamb momentum. As for the Lamb shift, the divergence of EM momentum is removed by mass renormalization [5], which eliminates the result obtained in Ref. [3]. What remains is a Lamb momentum of the order of $\alpha^{2}$ times the classical Abraham momentum [6,7]. Quantum vacuum momentum was also demonstrated for a chiral quantum particle in a magnetic field [8,9].

\footnotetext{
*bart.van-tiggelen@grenoble.cnrs.fr

Published by the American Physical Society under the terms of the Creative Commons Attribution 4.0 International license. Further distribution of this work must maintain attribution to the author(s) and the published article's title, journal citation, and DOI.
}

\section{ROTATING CHARGE COUPLED TO QUANTUM VACUUM}

Electromagnetic angular momentum is of great recent interest [10] and in macroscopic media even controversial [11], since matter and radiation are hard to disentangle. A vast amount of literature exists on Casimir torques [12-15]. On the nanoscale, these torques are caused by vacuum photons with energies up to $1 \mathrm{keV}$. In this paper we will show that the angular momentum of the quantum vacuum also emerges on a microscopic scale, though at much higher energies, like in the Lamb shift up to $1 \mathrm{MeV}$, the rest mass energy of the electron. The simplest microscopic model for which this problem can be solved is the well-known cyclotron problem of a nonrelativistic charge $q$ without spin rotating in a uniform magnetic field $\mathbf{B}_{0}$, in quantum mechanics better known as the Landau problem. We will show that, despite the nonrelativistic treatment, the angular momentum of the quantum vacuum is finite after mass renormalization, without the need for a cutoff in energy. While its proportionality to $q \mathbf{B}_{0}$ follows from invariance under $C, P$, and $T$ symmetry, and any additional dependence on the fine-structure constant is a standard QED outcome, the relation of angular momentum to the rotational energy of the charge is not a priori clear. In the symmetric Coulomb gauge and in Gaussian units, the vector potential $\mathbf{A}_{0}(\mathbf{r}, t)=\mathbf{B}_{0}(t) \times \mathbf{r} / 2$ conveniently preserves symmetry under rotations along the magnetic field. With the quantum vacuum, whose vector potential is also expressed in the Coulomb gauge $\boldsymbol{\nabla} \cdot \mathbf{A}=0$, the nonrelativistic Hamiltonian reads

$$
H=\frac{1}{2 \mu}\left(\mathbf{p}-\frac{q}{c_{0}} \mathbf{A}_{0}(\mathbf{r}, t)-\frac{q}{c_{0}} \mathbf{A}(\mathbf{r})\right)^{2}+\sum_{k \Pi} \hbar \omega_{k} a_{k \Pi}^{\dagger} a_{k \Pi} .
$$

Here $\mathbf{p}$ is the canonical momentum satisfying $\left[r_{i}, p_{j}\right]=i \hbar \delta_{i j}$. It is customary to choose the $z$ axis along the direction of $q \mathbf{B}_{0}$, which is charge invariant like the angular momentum, and to introduce the cyclotron frequency $\omega_{c}=|q| B_{0} / \mu c_{0}$. We have left out the quantum vacuum energy $\sum_{k \Pi} \hbar \omega_{k} / 2$ that represents here vacuum photons with constant infinite energy and zero angular momentum. It is convenient to exploit rotational symmetry as well for the quantum vacuum and express the vector potential of the quantum vacuum in terms of the spherical vector harmonics $\Phi_{\Pi}(\hat{\mathbf{k}})$ defined on the unit 
sphere in reciprocal space [16],

$$
\begin{aligned}
\mathbf{A}(\mathbf{r})= & \sqrt{2 \pi \hbar c_{0}} \sum_{\mathbf{k} \Pi} \frac{1}{k^{3 / 2}}\left[a_{k \Pi} \exp (-i \mathbf{k} \cdot \mathbf{r}) \Phi_{\Pi}(\hat{\mathbf{k}})\right. \\
& \left.+a_{k \Pi}^{\dagger} \exp (i \mathbf{k} \cdot \mathbf{r}) \bar{\Phi}_{\Pi}(\hat{\mathbf{k}})\right] .
\end{aligned}
$$

The continuum limit has been taken, $\sum_{\mathbf{k}} \equiv \int d^{3} \mathbf{k} /(2 \pi)^{3}$, and $\left[a_{k \Pi}, a_{k^{\prime} \Pi^{\prime}}^{\dagger}\right]=\delta_{k k^{\prime}} \delta_{\Pi, \Pi^{\prime}}$. The vector index $\Pi=\{J, M, p\}$ summarizes the three discrete quantum numbers of total EM angular momentum $J$, its $z$ component $M$, and two transverse polarizations $p$, the longitudinal vector harmonic being excluded by the Coulomb gauge. Without the quantum vacuum, the kinetic momentum of the charge is $\mathbf{p}^{K}=\mu d \mathbf{r} / d t=$ $\mu\left[H_{0}, \mathbf{r}\right] / i \hbar=\mathbf{p}-q \mathbf{A}_{0}(\mathbf{r}, t) / c_{0}$. In the Coulomb gauge, the canonical angular momentum $l_{z}=(\mathbf{r} \times \mathbf{p})_{z}$ commutes with $H_{0}$ and has eigenvalues $(|m|-n) \hbar$. This quantity is however gauge variant and any observable end result should be independent of $m$. The Hamiltonian $H_{0}$ has the eigenstates $\left|n, m, k_{z}\right\rangle$ of a two-dimensional harmonic oscillator whose energy levels $E_{n}=\left(n+\frac{1}{2}\right) \hbar \omega_{c}+\hbar^{2} k_{z}^{2} / 2 \mu$ are indeed independent of $m$ due to gauge invariance. Note that the Coulomb gauge is highly adapted to the present problem of cyclotron motion of a charge, but much less to the quantum Hall effect [17].

In the Coulomb gauge, total canonical angular momentum $\mathcal{L}_{z}$ is conserved, equal to the sum of the canonical angular momentum $l_{z}$ of the charge and the angular momentum of the transverse EM field $J_{z}$. The angular kinetic momentum operator of the charge, which is gauge-invariant, is not conserved and can be written as

$$
l_{z}^{K}=\mathcal{L}_{z}-J_{z}-\frac{q}{c_{0}}(\mathbf{r} \times \mathbf{A})_{z}-l^{L} .
$$

The Lenz term $l^{L}=\mu \omega_{c} \rho^{2} / 2$ is responsible for the electromotive force in classical electrodynamics, recently discussed quantum mechanically [18], and is a key element in the EM momentum controversy [19]. Without the quantum vacuum, the kinetic angular momentum of a Landau state is given by $\left\langle n, m\left|l_{z}^{K}\right| n, m\right\rangle=-(2 n+1) \hbar=-2 E_{n}($ rot $) / \omega_{c}$, which again is independent of $m$. When the quantum vacuum is included, $\left\langle l_{z}^{K}\right\rangle$ will decay radiatively, but slow enough for $\left\langle l_{z}^{K}\right\rangle$ to be well defined and modified.

We will use time-dependent perturbation theory to calculate the different contributions of the quantum vacuum to $l_{z}^{K}$ for an excited Landau state, all proportional to $q B_{0}(t)$ for slow adiabatic changes. We identify the interaction $W=$ $-\left(q / \mu c_{0}\right) \mathbf{p}^{K} \cdot \mathbf{A}(\mathbf{r})$ between rotating charge and quantum vacuum and imagine switching it on slowly like $W(t)=$ $W \exp (\epsilon t / \hbar)$ at $t_{0} \rightarrow-\infty$, when the total wave function is assumed to be in the pure state $|N\rangle=\left|n, m, k_{z}=0\right\rangle \otimes|\{0\}\rangle$ [20]. In this processus $\mathcal{L}_{z}$ is conserved and equal to its initial value $(|m|-n) \hbar$. The index $N^{\prime}$ refers to all possible product states $\left\{n, m, k_{z}\right\} \otimes\left\{n_{k} \Pi\right\}$ of charge plus transverse photons. Explicit reference will not be made to the highly degenerated levels $m$ or to the momentum $k_{z}$ of the charge along the magnetic field. The degeneration of the $m$ levels is protected by gauge invariance, and the impact of photon recoil on the longitudinal displacement is negligible. Since $W_{N N}=0$, the wave function at $t \geqslant t_{0}$ is perturbed as

$$
\begin{aligned}
\left|\Psi_{N}(t)\right\rangle= & \exp \left(-\frac{i}{\hbar} \int_{t_{0}}^{t} d t^{\prime}\left[E_{N}+\Delta E_{N}\left(t^{\prime}\right)\right]\right)|N\rangle \\
& +\sum_{N^{\prime}}^{\prime}\left|N^{\prime}\right\rangle \frac{W_{N^{\prime} N}(t)}{E_{N}-E_{N^{\prime}}+i \epsilon}+\cdots,
\end{aligned}
$$

with $\Delta E_{N}(t)=\sum_{N^{\prime}}^{\prime} W_{N N^{\prime}}(t) W_{N^{\prime} N}(t) /\left(E_{N}-E_{N^{\prime}}+i \epsilon\right)$ the second-order perturbation of the energy level $E_{N}$, the sum $\sum_{N^{\prime}}^{\prime}$ avoiding the initial level $N .{ }^{1}$ For an excited state, $\int_{t_{0}}^{t} d t^{\prime} \Delta E_{N}\left(t^{\prime}\right)=\int_{t_{0}}^{t} d t^{\prime}\left(E_{n}^{L}+\hbar A_{n} / 2 i\right)-\frac{1}{2} i \hbar \mathcal{N}_{n}$, with $\mathcal{N}_{n}$ a time-independent normalization of the wave function. The Lamb shift and spontaneous emission rate are, respectively,

$$
E^{L}=\hbar \omega_{c} \frac{2 \alpha}{3 \pi} x \ln \frac{2}{x}, \quad A_{n}=n \omega_{c} \frac{4 \alpha}{3} x,
$$

with $x=\hbar \omega_{c} / \mu c_{0}^{2}$ and $\alpha=q^{2} / \hbar c_{0}$ the fine-structure constant; $E^{L}$ is equal for all Landau levels, whereas radiative decay is proportional to rotational energy.

\section{ANGULAR MOMENTUM OF THE QUANTUM VACUUM}

In reciprocal space, the transverse EM momentum $J_{z}$ in Eq. (3) is expressed as $\left(J_{z}\right)(\mathbf{k})_{i j}=-i \hbar \delta_{i j}\left(\mathbf{k} \times \nabla_{\mathbf{k}}\right)_{z}-i \hbar \epsilon_{z i j}$, i.e., as a sum of orbital angular momentum and spin [16]. Neither one of them behaves as a genuine angular momentum [21], but this separation is physically useful. In Hilbert space $J_{z}$ reads

$$
J_{z}=\sum_{\mathbf{k}} \alpha_{i}^{\dagger}(\mathbf{k}) J_{i j}(\mathbf{k}) \alpha_{j}(\mathbf{k}),
$$

with the photon annihilation operator $\alpha_{i}(\mathbf{k})=$ $k^{-1} \sum_{\Pi} a_{k \Pi} \Phi_{\Pi, i}(\hat{\mathbf{k}})$ and its associated creation operator $\alpha_{i}^{\dagger}(\mathbf{k})$. The quantum expectation of $J_{z}$ is obtained by inserting the linearly perturbed eigenfunction (4) on both sides of the matrix element $\left\langle\Psi_{N}(t)\left|J_{z}\right| \Psi_{N}(t)\right\rangle$. This creates either a virtual or a real photon with energy $\hbar \omega_{k}$ and angular momentum $\boldsymbol{\Pi}^{\prime \prime}$ out of the quantum vacuum at the cost of canonical angular momentum of the charge. Working out the photon operators leaves us with

$$
\begin{aligned}
\left\langle J_{z}\right\rangle= & \frac{2 \pi \hbar q^{2}}{\mu^{2} c_{0}} e^{2 \epsilon t} \sum_{\mathbf{k}} \frac{1}{k^{2}} \sum_{\mathbf{k}^{\prime} \boldsymbol{\Pi}^{\prime} \mathbf{k}^{\prime \prime} \boldsymbol{\Pi}^{\prime \prime}} \frac{\delta_{k k^{\prime}} \delta_{k^{\prime \prime} k}}{\left(k^{\prime}\right)^{3 / 2}\left(k^{\prime \prime}\right)^{3 / 2}} \\
& \times\langle n| \mathbf{p}^{K} \cdot \boldsymbol{\Phi}_{\Pi^{\prime}}\left(\hat{\mathbf{k}}^{\prime}\right) e^{i \mathbf{k}^{\prime} \mathbf{r}} \frac{1}{E_{n}-H_{0}^{\prime}-\hbar \omega_{k}-i \epsilon} \\
& \times \overline{\boldsymbol{\Phi}}_{\Pi^{\prime} i}(\hat{\mathbf{k}}) J_{i j}(\mathbf{k}) \boldsymbol{\Phi}_{\Pi^{\prime \prime} j}(\hat{\mathbf{k}}) \\
& \times \frac{1}{E_{n}-H_{0}^{\prime}-\hbar \omega_{k}+i \epsilon} \mathbf{p}^{K} \cdot \overline{\boldsymbol{\Phi}}_{\Pi^{\prime \prime}}\left(\hat{\mathbf{k}}^{\prime \prime}\right) e^{-i \mathbf{k}^{\prime \prime} \mathbf{r}}|n\rangle .
\end{aligned}
$$

In principle, $\Pi^{\prime}=\Pi^{\prime \prime}$ since the spherical harmonics are orthogonal eigenfunctions of $J_{i j}(\mathbf{k})$. The mathematics is easier by using their completeness $\sum_{\Pi} \overline{\boldsymbol{\Phi}}_{\Pi}(\hat{\mathbf{k}}) \boldsymbol{\Phi}_{\Pi}\left(\hat{\mathbf{k}}^{\prime}\right)=\delta_{\hat{\mathbf{k}} \hat{\mathbf{k}}^{\prime}} \Delta(\hat{\mathbf{k}})$, with $\Delta(\hat{\mathbf{k}})$ transverse to $\hat{\mathbf{k}}$ imposed by the Coulomb gauge.

\footnotetext{
${ }^{1}$ We ignored the perturbation $\left|\delta \Psi_{N}\right\rangle=\sum_{N^{\prime} N^{\prime \prime}}^{\prime}\left|N^{\prime}\right\rangle W_{N^{\prime} N^{\prime \prime}} W_{N^{\prime \prime} N} /$ $\left(E_{N N^{\prime}}+2 i \epsilon\right)\left(E_{N N^{\prime \prime}}+i \epsilon\right)$. For one-photon processes, the matrix element $\left\langle n,\{0\}\left|l_{z}^{K}\right| \delta \Psi_{n\{0\}}\right\rangle$ vanishes.
} 
The exponential $\exp (i \mathbf{k} \cdot \mathbf{r})$ can be moved by using the operator identity $\exp (i \mathbf{k} \cdot \mathbf{r}) f(\mathbf{p})=f(\mathbf{p}-\hbar \mathbf{k}) \exp (i \mathbf{k} \cdot \mathbf{r})$, which induces a photon recoil in $H_{0}(\mathbf{p})$. With $\delta_{\mathbf{k k}^{\prime}}=\delta_{\hat{\mathbf{k}} \hat{\mathbf{k}}^{\prime}} \delta_{k k^{\prime}} / k^{2}$,

$$
\begin{aligned}
\left\langle J_{z}\right\rangle= & \frac{2 \pi \hbar e^{2 \epsilon t / \hbar} q^{2}}{\mu^{2} c_{0}} \sum_{\mathbf{k} \mathbf{k}^{\prime \prime}}\langle n| p_{m}^{K} \frac{1}{E_{n}-H_{0}^{\prime}(\mathbf{p}-\hbar \mathbf{k})-\hbar \omega_{k}-i \epsilon} \\
& \times \frac{1}{k} e^{i \mathbf{k}^{\prime} \cdot \mathbf{r}} \Delta_{m i}(\hat{\mathbf{k}}) J_{i j}(\mathbf{k}) \Delta_{j l}\left(\hat{\mathbf{k}}^{\prime \prime}\right) e^{-i \mathbf{k}^{\prime \prime} \cdot \mathbf{r}} \delta_{\mathbf{k} \mathbf{k}^{\prime \prime}} \\
& \times \frac{1}{E_{n}-H_{0}^{\prime}\left(\mathbf{p}-\hbar \mathbf{k}^{\prime \prime}\right)-\hbar \omega_{k}+i \epsilon} p_{l}^{K}|n\rangle .
\end{aligned}
$$

From this expression the EM spin of the quantum vacuum can be identified as

$$
\begin{aligned}
\left\langle S_{z}\right\rangle= & \frac{1}{3} \frac{2 \pi \hbar e^{2 \epsilon t / \hbar} q^{2}}{\mu^{2} c_{0}} \frac{\hbar}{i} \epsilon_{z i j} \\
& \times \sum_{\mathbf{k}} \frac{1}{k}\left\langle n\left|p_{i}^{K} \frac{1}{\left|E_{n}-H_{0}^{\prime}-\mathcal{E}(k)+i \epsilon\right|^{2}} p_{j}^{K}\right| n\right\rangle .
\end{aligned}
$$

We have performed the angular integral over $\mathbf{k}$ to eliminate $\Delta(\hat{\mathbf{k}})$, neglected the photon recoil $\mathbf{p}^{K} \cdot \hbar \mathbf{k} / \mu$ irrelevant for spin, and defined the energy $\mathcal{E}(k)=\hbar \omega_{k}+\hbar^{2} k^{2} / 2 \mu$. The orbital angular momentum is associated with a differential operator acting on $\delta_{\mathbf{k k}^{\prime \prime}}$, and an integration by parts is imposed to perform the integral over $\mathbf{k}^{\prime \prime}$. Since this operator acts only on angles, we find, for the orbital angular momentum,

$$
\begin{aligned}
\left\langle L_{z}\right\rangle= & \frac{2 \pi \hbar e^{2 \epsilon t / \hbar} q^{2}}{\mu^{2} c_{0}} \sum_{\mathbf{k}}\langle n| p_{m}^{K} \frac{\hbar}{i} \epsilon_{z s t} \\
& \times \hat{k}_{t} \nabla_{s}\left(\Delta_{m i}(\hat{\mathbf{k}}) \frac{1}{E_{n}-H_{0}^{\prime}(\mathbf{p}-\hbar \mathbf{k})-\hbar \omega_{k}-i \epsilon} e^{i \mathbf{k} \cdot \mathbf{r}}\right) \\
& \times \Delta_{i l}(\hat{\mathbf{k}}) e^{-i \mathbf{k} \cdot \mathbf{r}} \frac{1}{E_{n}-H_{0}^{\prime}(\mathbf{p}-\hbar \mathbf{k})-\hbar \omega_{k}+i \epsilon} p_{l}^{K}|n\rangle .
\end{aligned}
$$

As $k_{z}=0$, the kinetic operator $\mathbf{p}^{K}$ is located in the $x y$ plane. It is customary to write $p_{x}^{K}+(-) i p_{y}^{K}=\left(2 \mu \hbar \omega_{c}\right)^{1 / 2} c^{(\dagger)}$ in terms of the raising and lowering operators of the Landau levels, in terms of which $H_{0}=\hbar \omega_{c}\left(c^{\dagger} c+\frac{1}{2}\right)$. To evaluate the spin in Eq. (6) we use $\epsilon_{z i j} p_{i}^{K} f\left(H_{0}\right) p_{j}^{K}=-i \mu \hbar \omega_{c}\left[c^{\dagger} f\left(E_{n-1}\right) c-\right.$ $\left.c f\left(E_{n+1}\right) c^{\dagger}\right]$. The first term implies the release of a real photon with energy $\hbar \omega_{c}$. As $\epsilon \downarrow 0$, this part of $\left\langle S_{z}\right\rangle$ is written as $(\hbar / 2) \int_{t_{0}}^{t} d t^{\prime} \exp \left(2 \epsilon t^{\prime} / \hbar\right) \delta\left(\hbar \omega_{c}-\hbar \omega_{k}\right)$, so

$$
\frac{d}{d t}\left\langle S_{z}\right\rangle=-\frac{1}{2} A_{n} \hbar,
$$

with $A_{n}$ defined in Eq. (4). The second term involves virtual photons and is finite as $\epsilon \downarrow 0$,

$$
\begin{aligned}
\left\langle S_{z}\right\rangle & =(n+1) \frac{q^{2} \hbar^{3} \omega_{c}}{3 \mu c_{0}} \int_{0}^{\infty} \frac{k d k}{\left[\hbar \omega_{c}+\mathcal{E}(k)\right]^{2}} \\
& =\alpha \frac{\hbar}{3 \pi}(n+1) x \ln \frac{2}{x} .
\end{aligned}
$$

In expression (7) for $\left\langle L_{z}\right\rangle$ the derivative $\nabla_{\mathbf{k}}$ acts on the three factors inside large parentheses. Its action on the factor in the middle, caused by the photon recoil, is a factor $x$ smaller than the rest. The action on the first factor gives $\epsilon_{z s t} \hat{k}_{t} \nabla_{s}\left(\Delta_{m i}\right) \Delta_{i l}=-k^{-1} \epsilon_{z l t} \hat{k}_{m} \hat{k}_{t}$ and produces an an- gular momentum $\left\langle L_{z}^{(1)}\right\rangle=\left\langle S_{z}\right\rangle$. Finally, the action of $\nabla_{\mathbf{k}}$ on $\exp (i \mathbf{k} \cdot \mathbf{r})$ leads to the expression

$$
\begin{aligned}
\left\langle L_{z}^{(2)}\right\rangle= & \frac{2 \pi \hbar^{2} e^{2 \epsilon t / \hbar} q^{2}}{\mu^{2} c_{0}} \\
& \times \sum_{\mathbf{k}}\left\langle n\left|p_{m}^{K} \Delta_{m l}(\hat{\mathbf{k}}) \frac{1}{\mathcal{H}_{n}-i \epsilon}(\mathbf{r} \times \hat{\mathbf{k}})_{z} \frac{1}{\mathcal{H}_{n}+i \epsilon} p_{l}^{K}\right| n\right\rangle,
\end{aligned}
$$

where $\mathcal{H}_{n} \equiv E_{n}-H_{0}-\mathcal{E}(k)+\hbar \mathbf{p}^{K} \cdot \mathbf{k} / \mu$. This time, the photon recoil cannot be ignored and we must expand either one of the denominators which produces an integral $d k \hat{d} \mathbf{k}$ with integrand of the type

$$
\epsilon_{z s t} \Delta_{m l}(\hat{\mathbf{k}}) k_{u} \hat{k}_{t} p_{m}^{K} \frac{1}{\mathcal{H}_{n}-i \epsilon} p_{u}^{K} \frac{1}{\mathcal{H}_{n}-i \epsilon} r_{s} \frac{1}{\mathcal{H}_{n}+i \epsilon} p_{l}^{K} .
$$

Physically, this corresponds to the creation of a virtual photonic mode with finite orbital angular momentum. In terms of the infinitely degenerated center $(X, Y)$ of the cyclotron orbit, we associate $x=X-p_{y}^{K} / \mu \omega_{c}$ and $y=Y+p_{x}^{K} / \mu \omega_{c}$. The operators $(X, Y)$ drop out in the vacuum expectation value since they do not occur in pairs. Upon expressing $\left(p_{x}^{K}, p_{y}^{K}\right)$ in the operators $c$ and $c^{\dagger}$, the integrand contains four transition operators. As was the case for $\left\langle S_{z}\right\rangle$, some contribute to spontaneous emission but are seen to be a factor $x$ smaller than $A_{n}$. We thus focus on terms where the limit $\epsilon \downarrow 0$ exists. For instance, the sequence

$$
\left\langle n\left|c \mathcal{H}_{n}^{-1} c^{\dagger} \mathcal{H}_{n}^{-1} c \mathcal{H}_{n}^{-1} c^{\dagger}\right| n\right\rangle=\frac{(n+1)(n+2)}{\mathcal{E}(k)^{3}}
$$

leads to

$$
\begin{aligned}
\left\langle L_{z}^{(2)}\right\rangle & \sim(n+1)(n+2) \frac{2 \pi \hbar^{2} q^{2}}{\mu^{2} c_{0}} \frac{\hbar}{\mu} \mu \hbar^{2} \omega_{c} \sum_{\mathbf{k}} \frac{k}{\mathcal{E}(k)^{3}} \\
& \approx \frac{\hbar}{8 \pi} \alpha(n+1)(n+2) x .
\end{aligned}
$$

Upon collecting all possibilities, performing the angular integral, and adding the complex conjugate, we obtain

$$
\left\langle J_{z}\right\rangle=\left\langle S_{z}\right\rangle-\frac{4 \hbar}{15 \pi} \alpha(n+1)(n+4) x
$$

and $d / d t\left\langle J_{z}\right\rangle=-\hbar A_{n} / 2$.

The penultimate term in Eq. (3), the longitudinal EM angular momentum $\Delta L_{\|}$, is itself linear in the vacuum field. Its leading quantum expectation value is obtained using the linear perturbation of the eigenfunctions and the completeness of the spherical vector harmonics

$$
\left\langle\Psi_{N}\left|\Delta L_{\|}\right| \Psi_{N}\right\rangle=-\frac{q^{2}}{\mu c_{0}} \frac{4 \pi \hbar}{3} \sum_{\mathbf{k}} \frac{1}{k} \epsilon_{z i j}\left\langle n\left|r_{i} \frac{1}{\mathcal{H}_{n}+i \epsilon} p_{j}^{K}\right| n\right\rangle
$$

plus its complex conjugate. Here the $k$ integral diverges in the UV as $d k / k$. We recognize for large $k$ the form $(\delta \mu / \mu)\left\langle n\left|l_{z}^{K}\right| n\right\rangle$, with $\delta \mu$ the well-known Bethe-Kramers mass renormalization [1]. Upon adding it to the kinetic mass as $\mathbf{I}^{K}=(\mu+\delta \mu) \mathbf{r} \times d \mathbf{r} / d t$ and subtracting it from the above 
equation we obtain

$$
\begin{aligned}
\left\langle\Psi_{N}\left|\Delta L_{\|}\right| \Psi_{N}\right\rangle= & -\frac{q^{2}}{\mu c_{0}} \frac{4 \pi \hbar}{3} \sum_{\mathbf{k}} \frac{1}{k} \sum_{n^{\prime}}^{\prime} \frac{i \hbar}{\mu} \\
& \times \epsilon_{i j z} \frac{\left\langle n\left|p_{i}^{K}\right| n^{\prime}\right\rangle}{\Delta E_{n^{\prime} n}+\mathcal{E}(k)-i \epsilon} \frac{\left\langle n^{\prime}\left|p_{j}^{K}\right| n\right\rangle}{\mathcal{E}(k)}+\text { c.c., }
\end{aligned}
$$

where we have used the identity $\Delta E_{n^{\prime} n}\left\langle n|\mathbf{r}| n^{\prime}\right\rangle=$ $(i \hbar / \mu)\left\langle n\left|\mathbf{p}^{K}\right| n^{\prime}\right\rangle$. The operators $p_{x}^{K}$ and $p_{y}^{K}$ can be expressed in terms of $c^{\dagger}$ and $c$, which results in

$$
\left\langle\Delta L_{\|}\right\rangle=\hbar \frac{4 \alpha}{3 \pi} x \ln \frac{2}{x},
$$

which, like the Lamb shift in energy, is independent of $n$.

The last contribution of the quantum vacuum to the angular momentum is associated with the Lenz term $l^{T}$ in Eq. (3). The quantum vacuum comes in via $\mathcal{N}_{n}$ and the second term in Eq. (4). With $l^{T}=\hbar\left(c^{\dagger} c+1+b^{\dagger} b-i b^{\dagger} c^{\dagger} / 2+i c b / 2\right)$ in terms of the raising $\left(b^{\dagger}\right)$ and lowering $(b)$ operator of the degenerated $m$ levels [17], the $m$ dependence is seen to cancel in the sum of both terms. The second term equals $\hbar(n+1)$ times

$$
\begin{gathered}
\frac{4 \pi \hbar q^{2}}{3 \mu^{2} c_{0}} \frac{1}{i \hbar} \int_{t_{0}}^{t} d t^{\prime} e^{2 \epsilon t^{\prime} / \hbar} \sum_{\mathbf{k}}\left\langle n\left|p_{j}^{K} \frac{1}{\mathcal{H}_{n}+i \epsilon} p_{j}^{K}\right| n\right\rangle+\text { c.c. } \\
=-A_{n} \int_{t_{0}}^{t} d t^{\prime} e^{2 \epsilon t^{\prime} / \hbar}-\frac{2 \alpha}{3 \pi}(n+1) x \ln \frac{2}{x}
\end{gathered}
$$

and the first is $\hbar$ times

$$
\begin{gathered}
\frac{4 \pi \hbar q^{2}}{3 \mu^{2} c_{0}} e^{2 \epsilon t / \hbar} \sum_{\mathbf{k}} \frac{1}{k}\left\langle n\left|p_{j}^{K} \frac{1}{\mathcal{H}_{n}+i \epsilon}\left(c^{\dagger} c+1\right) \frac{1}{\mathcal{H}_{n}-i \epsilon} p_{j}^{K}\right| n\right\rangle \\
=n A_{n} \int_{t_{0}}^{t} d t^{\prime} e^{2 \epsilon t^{\prime} / \hbar}+\frac{2 \alpha}{3 \pi}(n+1)(n+2) x \ln \frac{2}{x} .
\end{gathered}
$$

In particular, $n^{2}$ terms also cancel in the sum and

$$
\left\langle\delta l^{T}\right\rangle=2\left\langle S_{z}\right\rangle, \quad \frac{d}{d t}\left\langle\delta l^{T}\right\rangle=-\hbar A_{n} .
$$

By adding up the four contributions $\left\langle S_{z}\right\rangle,\left\langle J_{z}\right\rangle,\left\langle J_{\|}\right\rangle$, and $\left\langle\delta l^{T}\right\rangle$ we find, for the total angular momentum of the quantum vacuum,

$$
\left\langle J_{z}^{Q V}\right\rangle=\hbar \frac{4 \alpha}{3 \pi}(n+2) x \ln \frac{2}{x}-\hbar \frac{4 \alpha}{15 \pi}(n+1)(n+4) x
$$

and $d\left\langle J_{z}^{Q V}\right\rangle / d t=-2 \hbar A_{n}$. We conclude that the quantum vacuum achieves an angular momentum directed along the $q \mathbf{B}_{0}$ axis and is, in units of $\hbar$, proportional to $\alpha \times x=\alpha \times$ $\hbar \omega_{c} / \mu c_{0}^{2} \approx 10^{-12}$ per Tesla, which is time dependent if $B_{0}$ is. Not unsurprisingly, for a rapidly rotating charge (large $n$ ), the orbital angular momentum of the quantum vacuum, expressed by the second term, dominates over the spin component. In all momentum integrals the photon momentum $\hbar k$ takes values up to $\mu c_{0}$ with nonetheless a significant weight of nonrelativistic momenta. A relativistic description of the rotating electron is thus relevant but should affect only numerical coefficients in Eq. (13). Even in a relativistic picture, Eq. (3) for the kinetic angular momentum is valid and $\left\langle l_{z}^{K}\right\rangle$ remains quantized to $-(2 n+1) \hbar .^{2}$ In the ground state, the existence of Lamb angular momentum makes the kinetic angular momentum slightly more negative than $-\hbar$; in states with large $n$ it will be slightly less negative than $-(2 n+1) \hbar$, the correction growing like $n^{2}$. Note that the gauge-invariant magnetic moment $M_{z}=(q / 2 \mu) l^{K}$ of the rotating charge is subject to the same correction. Due to the quantum vacuum, the kinetic angular momentum $-(2 n+1) \hbar$ of the charge in Landau level $n$ decays to the Landau level $n-1$ with rate $A_{n}$, so we expect that $d\left\langle l^{K}\right\rangle / d t=+2 \hbar A_{n}$. Hence, $\left\langle l_{z}^{K}+J_{z}^{Q V}\right\rangle=$ $\mathcal{L}_{z}$ is conserved in the decay. This spontaneous emission could be seen as the most elementary form of rotational quantum friction [22]. The nonrelativistic analysis imposes that $E_{n} \ll \mu c_{0}^{2}$, implying, for an electron in a field of $10 \mathrm{~T}$, that $n \ll 10^{9}$. Pushing our theory to this extreme synchrotron regime, the relative contribution of Lamb orbital angular momentum would be of order $10^{-4}$.

\section{CONCLUSION}

The main objective of this work was to establish the existence of angular momentum of the EM quantum vacuum, induced by the presence of a rotating charge in a magnetic field. It is instructive to look at the separate contributions of spin, orbital angular momentum, and angular momentum directly associated with the gauge fields. All are oriented along the magnetic field and proportional to the product of the fine-structure constant and the small ratio of rotational energy to rest energy. Spin and orbital angular momentum decay in the same way, their coupling being large, yet the orbital angular momentum of the quantum vacuum, induced by photon recoil, dominates angular momentum for highly energetic Landau levels. Similar to the Lamb shift in energy, this angular Lamb momentum is governed by virtual photons with energies up to the rest mass of the charge and, despite the UV renormalizability of the theory, would merit a relativistic treatment. A future challenge would be to study EM angular momentum in the fully relativistic synchrotron problem or to investigate it for Rydberg orbits.

\footnotetext{
${ }^{2}$ The eigenfunctions are the same for the relativistic Hamiltonian without spin, and thus the Lenz moment $\left\langle l^{T}\right\rangle$ remains unaltered, which in turn determines $l_{z}^{K}[16]$.
}

[1] P. W. Milonni, The Quantum Vacuum (Academic Press, San Diego, 1994).
[2] K. A. Milton, The Casimir Effect (World Scientific, Singapore, 2001); M. Bordag, G. L. Klimchitskaya, U. Mohideen, and 
V. M. Mostepanenko, Advances in the Casimir Effect (Oxford University Press, New York, 2009).

[3] A. Feigel, Phys. Rev. Lett. 92, 020404 (2004).

[4] B. A. van Tiggelen, Eur. Phys. J. D 73, 196 (2019).

[5] B. A. van Tiggelen, S. Kawka, and G. L. J. A. Rikken, Eur. Phys. J. D 66, 272 (2012).

[6] R. Loudon, Fortschr. Phys. 52, 1134 (2004).

[7] G. L. J. A. Rikken and B. A. van Tiggelen, Phys. Rev. Lett. 107, 170401 (2011).

[8] M. Donaire, B. A. van Tiggelen, and G. L. J. A. Rikken, Phys. Rev. Lett. 111, 143602 (2013).

[9] M. Donaire, J. High Energy Phys. 10 (2019) 041.

[10] K. Y. Bliokh and F. Nori, Phys. Rep. 592, 1 (2015).

[11] M. Kristensen and J. P. Woerdman, Phys. Rev. Lett. 72, 2171 (1994).

[12] S. J. van Enk, Phys. Rev. A 52, 2569 (1995).

[13] F. Capasso, J. N. Munday, D. Iannuzzi, and H. B. Chan, IEEE J. Sel. Top. Quantum Electron. 13, 400 (2007).

[14] T. G. Philbin and U. Leonhardt, Phys. Rev. A 78, 042107 (2008).
[15] F. Impens, A. M. Contreras-Reyes, P. A. Maia Neto, D. A. R. Dalvit, R. Guérout, A. Lambrecht, and S. Reynaud, Europhys. Lett. 92, 40010 (2010).

[16] C. Cohen-Tannoudji, J. Dupont-Roc, and G. Grynberg, Photons and Atoms (Wiley, New York, 1989).

[17] M. O. Goerbig, in Ultracold Gases and Quantum Information, edited by C. Miniatura, L.-C. Kwek, M. Ducloy, B. Grémaud, B.-G. Englert, L. Cugliandolo, A. Ekert, and K. K. Phua, Proceedings of the Les Houches Summer School, XCI, 2009 (Oxford University Press, Oxford, 2011).

[18] C. R. Greenshields, R. L. Stamps, S. Franke-Arnold, and S. M. Barnett, Phys. Rev. Lett. 113, 240404 (2014).

[19] E. A. Hinds and S. M. Barnett, Phys. Rev. Lett. 102, 050403 (2009).

[20] R. Loudon, The Quantum Theory of Light (Oxford University Press, Oxford, 1991).

[21] S. van Enk and G. Nienhuis, Europhys. Lett. 25, 497 (1994).

[22] T. G. Philbin and U. Leonhardt, New J. Phys. 11, 033035 (2009); J. B. Pendry, ibid. 12, 033028 (2010). 